numerical modelling has been used to examine deformation in the lower mantle associated with downwelling of subducting slabs. It has been suggested that dislocation creep is expected to concentrate in these regions ${ }^{20}$. This deformation mechanism leads to the development of crystal preferred orientation (CPO), which is a major source of seismic anisotropy. In the absence of experimental data on the plastic deformation of magnesium silicate perovskite, previous studies have focused on the contribution of magnesiowustite only. The present study shows that dislocation creep can be activated in $\mathrm{MgSiO}_{3}$ perovskite at high temperature, contrary to what has been reported at room temperature ${ }^{21}$. Our results provide information on the geometry of slip in perovskite that is needed to model CPO in perovskite. However, forward modelling of seismic anisotropy requires the understanding of how strain partitions between the minor, weak phase and the major, hard phase of the magnesiowustite-perovskite assemblage. This could be achieved through highpressure/high-temperature deformation experiments such as those described here, or by three-dimensional modelling of plastic deformation of a two-phase polycrystalline aggregate.

Received 19 December 2003; accepted 5 March 2004; doi:10.1038/nature02472.

1. Ringwood, A. E. Phase transformations and their bearing on the constitution and dynamics of the mantle. Geochim. Cosmochim. Acta 55, 2083-2110 (1991).

2. Poirier, J. P., Peyronneau, J., Gesland, J. Y. \& Brebec, G. Viscosity and conductivity of the lower mantle; an experimental study on $\mathrm{MgSiO}_{3}$ perovskite analogue, $\mathrm{KZnF}_{3}$. Phys. Earth Planet. Inter. 32, 273-287 (1983).

. Beauchesne, S. \& Poirier, J. P. Creep of barium titanate perovskite: a contribution to a systematic approach to the viscosity of the mantle. Phys. Earth Planet. Inter. 55, 187-199 (1989).

4. Beauchesne, S. \& Poirier, J. P. In search of a systematics for the viscosity of perovskites: creep of potassium tantalate and niobate. Phys. Earth Planet. Inter. 61, 182-198 (1990).

5. Wang, Z. C., Karato, S. \& Fujino, K. High temperature creep of single crystal strontium titanate $\left(\mathrm{SrTiO}_{3}\right)$ - a contribution to creep systematics in perovskites. Phys. Earth Planet. Inter. 79, 299-312 (1993).

6. Li, P., Karato, S. I. \& Wang, Z. High-temperature creep in fine-grained polycrystalline $\mathrm{CaTiO}_{3}$, an analogue material of $(\mathrm{Mg}, \mathrm{Fe}) \mathrm{SiO}_{3}$ perovskite. Phys. Earth Planet. Inter. 95, 19-36 (1996).

7. Wang, Z. C., Dupas-Bruzek, C. \& Karato, S. High temperature creep of an orthorhombic perovskite$\mathrm{YAlO}_{3}$. Phys. Earth Planet. Inter. 110, 51-69 (1999).

8. Chen, J. H., Weidner, D. J. \& Vaughan, M. T. The strength of $\mathrm{Mg}_{0.9} \mathrm{Fe}_{0.1} \mathrm{SiO}_{3}$ perovskite at high pressure and temperature. Nature 419, 824-826 (2002).

9. Horiuchi, H., Ito, E. \& Weidner, D. J. Perovskite-type $\mathrm{MgSiO}_{3}$ : single-crystal X-ray diffraction study. Am. Mineral. 72, 357-360 (1987).

10. Cordier, P. \& Rubie, D. C. Plastic deformation of minerals under extreme pressure using a multi-anvil apparatus. Mater. Sci. Eng. A 309, 38-43 (2001).

11. Caglioti, G., Paoletti, A. \& Ricci, F. P. Choice of collimators for a crystal spectrometer for neutron diffraction. Nucl. Instrum. Methods 3, 223-228 (1958).

12. Krivoglaz, M. A. Theory of X-ray and Thermal Neutron Scattering by Real Crystals (Plenum, New York, 1969).

13. Krivoglaz, M. A. X-ray and Neutron Diffraction in Nonideal Crystals (Springer, Berlin, 1996).

14. Wilkens, $M$. The determination of density and distribution of dislocations in deformed single crystals from broadened x-ray diffraction profiles. Phys. Status Solidi 2, 359-370 (1970).

15. Kuzel, R. \& Klimanek, P. X-ray diffraction line broadening due to dislocations in non-cubic crystalline materials. 3. Experimental results for plastically deformed zirconium. J. Appl. Crystallogr. 22, 299-307 (1989).

16. Ungár, T. \& Borbély, A. The effect of dislocation contrast on $\mathrm{x}$-ray line broadening: a new approach to line profile analysis. Appl. Phys. Lett. 69, 3173-3175 (1996).

17. Ungár, T., Gubicza, J., Ribárik, G. \& Borbély, A. Crystallite size distribution and dislocation structure determined by diffraction profile analysis: principles and practical application to cubic and hexagonal crystals. J. Appl. Crystallogr. 34, 298-310 (2001).

18. Ribárik, G., Ungár, T. \& Gubicza, J. MWP-fit: a program for multiple whole-profile fitting of diffraction peak profiles by ab initio theoretical functions. J. Appl. Crystallogr. 34, 669-676 (2001).

19. Karato, S. I. Plasticity of $\mathrm{MgSiO}_{3}$ perovskite: the results of microhardness tests on single crystals. Geophys. Res. Lett. 17, 13-16 (1990).

20. McNamara, A. K., vanKeken, P. E. \& Karato, S. I. Development of anisotropic structure in the Earth's lower mantle by solid-state convection. Nature 416, 310-314 (2002).

21. Merkel, S. et al. Deformation of $\left(\mathrm{Mg}_{0.9}, \mathrm{Fe}_{0.1}\right) \mathrm{SiO}_{3}$ perovskite aggregates up to $32 \mathrm{GPa}$. Earth Planet. Sci. Lett. 209, 351-360 (2003).

Acknowledgements High-pressure experiments were performed at the Bayerisches Geoinstitut under the EU 'IHP-Access to Research Infrastructures' programme. P.C. benefited from a 'Congé thématique pour recherche' from the University of Lille and from continuous support from INSU ('Intérieur de la Terre' programme). T.U. and G.T. thank the Hungarian Science Foundation for supporting this work

Competing interests statement The authors declare that they have no competing financial interests.

Correspondence and requests for materials should be addressed to P.C.

(Patrick.Cordier@univ-lillel.fr).

\section{Competition and mutualism among the gut helminths of a mammalian host}

\author{
Joanne Lello ${ }^{1}$, Brian Boag ${ }^{2}$, Andrew Fenton ${ }^{3}$, lan R. Stevenson ${ }^{4}$ \\ \& Peter J. Hudson ${ }^{5}$
}

${ }^{1}$ CSIRO Livestock Industries, F.D. McMaster Laboratories, Locked Bag 1, PO Armidale, New South Wales 2350, Australia

${ }^{2}$ Birch Brae, Knapp, Perth \& Kinross PH14 9SW, UK

${ }^{3}$ Institute of Zoology, ZSL, Regents Park, London NW1 4RY, UK

${ }^{4}$ Department of Biological Sciences, University of Stirling, Stirling FK9 4LA, UK

${ }^{5}$ Department of Biology, 513 Mueller Laboratory, University Park, Penn State

University, Pennsylvania 16802, USA

Most animal species are infected with multiple parasite species; however, the role of interspecific parasite interactions in influencing parasite dynamics and shaping parasite communities has been unclear. Although laboratory studies have found evidence of cross-immunity, immunosuppression and competition $^{1-6}$, analyses of hosts in the field have generally concluded that parasite communities are little more than random assemblages $^{7-14}$. Here we present evidence of consistent interspecific interactions in a natural mammalian system, revealed through the analysis of parasite intensity data collected from a freeranging rabbit (Oryctolagus cuniculus) population, sampled monthly for a period of $23 \mathrm{yr}$. The wild rabbit plays host to a diverse gut helminth community ${ }^{15-17}$ that reflects the communities seen in other economically important domestic herbivores $^{18,19}$. These findings suggest that parasite interactions could have profound implications for the dynamics of parasite communities. The efficacy of parasite control programmes could be jeopardized if such interactions are not taken into account. In contrast, a clear understanding of such interactions may provide the basis for the development of more environmentally acceptable methods of parasite control.

The wild rabbit population in the United Kingdom is dominated by five gut helminths: the strongylid nematodes Graphidium strigosum (in the stomach) and Trichostrongylus retortaeformis (small intestine); the anoplocephaloid cestodes Mosgovoyia pectinata (small intestine) and Cittotaenia denticulata (small intestine); and the oxyurid nematode Passalurus ambiguus (large intestine and colon). Our objectives were to identify (1) whether interspecific interactions could be detected within this parasite community; (2) quantify the strengths of these interactions; (3) identify the putative mechanisms by which interactions may be mediated; and (4) propose the possible consequences of such interactions for future parasite control.

We used a combination of generalized linear modelling (GLM) and residual maximum likelihood (REML) linear mixed model analyses of parasite count data to test the null hypothesis that parasite interactions do not influence parasite numbers. We included aspects of the host biology and the external environment that if excluded would be most likely to create the appearance of interspecific interactions where none exist (for further details see Supplementary Information). All predictions are presented as the percentage difference between the predicted level of the dependent variable (after back-transformation) when the interacting species has a zero count, compared with the predicted level of the dependent variable (after back-transformation) when the interacting species is at its geometric mean (in concomitant infection).

According to the models, same-locale, downstream (anterior to posterior) and upstream (posterior to anterior) interactions occurred between the helminths. One significant $(P<0.001)$ inter- 
action was seen between two species in the same location in the gut, with the model predicting a positive effect of $C$. denticulata on the intensity of $T$. retortaeformis infection (Fig. la and Table 1). The geometric mean of $C$. denticulata when in a concomitant infection with $T$. retortaeformis was around two individuals, which was predicted to increase the number of T. retortaeformis by $51 \%$ relative to a rabbit harbouring no $C$. denticulata.

Trichostrongylus retortaeformis was subject to a predicted, positive downstream influence from $G$. strigosum $(P<0.001$; Fig. $1 \mathrm{~b}$ and Table 1). In concomitant infections, the geometric mean of 18 G. strigosum was predicted to increase the number of T. retortaeformis by $36 \%$. A more complex downstream interaction was seen with $M$. pectinata in the small intestine, which was predicted to have a positive effect in female $(P=0.003)$, but not male, hosts on the intensity of $P$. ambiguus in the large intestine/colon (Fig. 1c and Table 1). The geometric mean of three $M$. pectinata was predicted to increase the intensity of $P$. ambiguus by $163 \%$ relative to a host containing no $M$. pectinata.

The model predicted that the association between these two species also operated in the opposite, upstream direction, with $P$. ambiguus having a positive effect on $M$. pectinata. Again, this association was complicated by host sex, such that $P$. ambiguus increased the intensity of $M$. pectinata in male but not female rabbits $(P=0.008$; Fig. $1 \mathrm{~d}$ and Table 1$)$. The geometric mean of $P$. ambiguus (in concomitant infection) in male rabbits was 127 , which was predicted to increase $M$. pectinata numbers by $67 \%$ relative to hosts not infected with P. ambiguus. Mosgovoyia pectinata also had an upstream effect, reducing the intensity of $G$. strigosum in the stomach $(P=0.011$; Fig. 1e and Table 1$)$ by $19 \%$ on average. Finally, the presence of $T$. retortaeformis was predicted to affect negatively the numbers of $G$. strigosum in an upstream position $(P=0.018$; Fig. $1 \mathrm{f}$ and Table 1$)$. This effect was complicated by host mass (a proxy for age $\mathrm{e}^{20}$ ), such that the effect decreased as rabbit mass increased; however, in a rabbit of average mass $(1,590 \mathrm{~g}$ in concomitantly infected rabbits), $T$. retortaeformis was predicted to reduce G. strigosum numbers by $29 \%$.

Analyses indicate the existence of a network of interactions between these helminths (Fig. 2). In most cases, based on the biology of the parasites, we can postulate the likely mechanisms underlying these interactions. The observed same-locale or downstream interactions may be due to direct influences of one parasite on another through by-products, manipulation of gut physiology, competition or physical crowding. However, for upstream interactions, the most likely route would be interaction mediated through the host's immune system, although changes in gut physiology, for example $\mathrm{pH}$ change, cannot be entirely ruled out ${ }^{21}$.

The putative explanation for the observed interactions between G. strigosum and T. retortaeformis is cross-immunity between these species. Age-intensity curves for T. retortaeformis and G. strigosum (with mass used as a proxy for age) support previous findings ${ }^{22-24}$
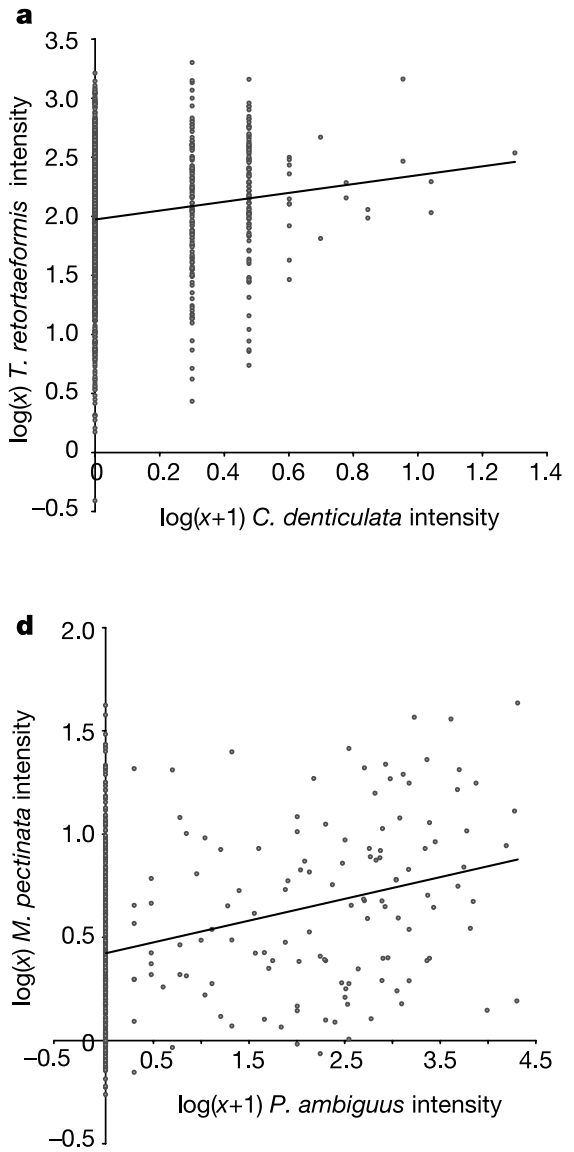

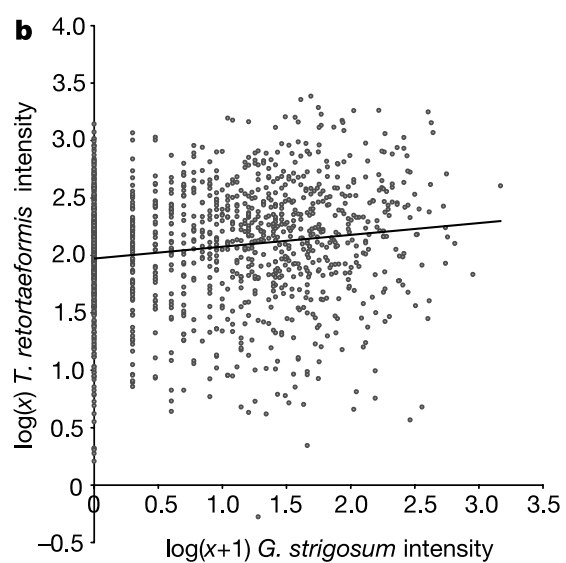

e

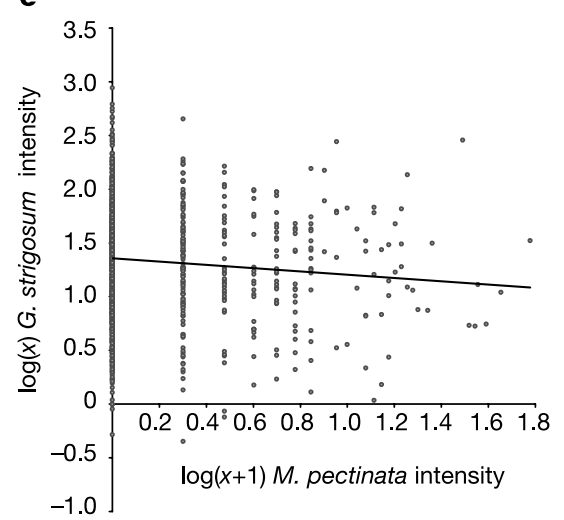

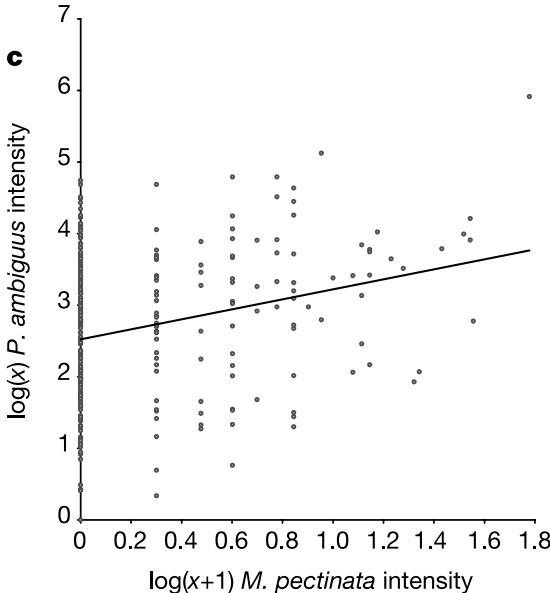

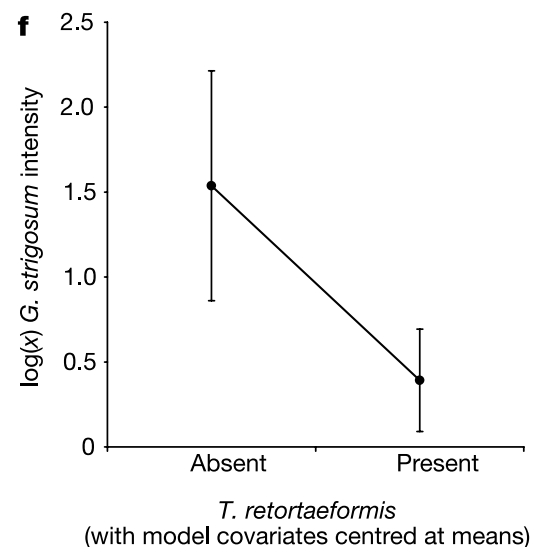

Figure 1 Predictions from generalized linear and restricted maximum likelihood models of the relationships between the dependent variable (affected species $\log (x)$ helminth intensity with zeros removed) and the independent variable $\mathbf{a}-\mathbf{e}$, effector species $\log (x+1)$ helminth intensity, or $\mathbf{f}$, effector species presence-absence data (in rabbits of average mass, $1,590 \mathrm{~g}$ ). All analyses were conducted on adult rabbit data. Lines of predicted fit and residual fit points (or 95\% confidence intervals) are shown in each case. Trichostrongylus retortaeformis with $C$. denticulata $(\mathbf{a}) ;$ T. retortaeformis with $G$. strigosum (b); P. ambiguus with M. pectinata (c); M. pectinata with $P$. ambiguus (d); G. strigosum with M. pectinata (e); and G. strigosum with $T$. retortaeformis (f). 


\begin{tabular}{|c|c|c|c|}
\hline Model term & $\begin{array}{c}\text { Test } \\
\text { statistic† }\end{array}$ & d.f. & $P$ value \\
\hline \multicolumn{4}{|l|}{$\log$ G. strigosum intensity } \\
\hline Month & 182.50 & 11 & $<0.00$ \\
\hline Host food type & 25.18 & 1 & $<0.001$ \\
\hline Presence/absence of myxomatosis & 8.84 & 1 & 0.018 \\
\hline T. retortaeformis*host mass $\log (x+1)$ M. pectinata & 6.6 & 1 & 0.011 \\
\hline \multicolumn{4}{|l|}{$\log M$. pectinata intensity§ } \\
\hline Month & 62.49 & 11 & $<0.00$ \\
\hline Sex & 0.00 & 1 & 0.95 \\
\hline Sex*log $(x+1) P$. ambiguus & 7.14 & 1 & 0.0 \\
\hline \multicolumn{4}{|l|}{$\log T$. retortaeformis intensityll } \\
\hline Month*host sex & 31.5 & 11,1195 & $<0.00$ \\
\hline Host sex*host mass & 2.8 & 1,1195 & 0.00 \\
\hline Presence/absence of myxomatosis & 12.8 & 1,1195 & $<0.00$ \\
\hline $\log (x+1)$ C. denticulata & 23.98 & 1,1195 & $<0.00$ \\
\hline $\log (x+1)$ G. strigosum & 31.94 & 1,1195 & $<0.00$ \\
\hline \multicolumn{4}{|l|}{$\log$ C. denticulata intensity\|l } \\
\hline Month & 1.41 & 11,288 & $<0.00$ \\
\hline \multicolumn{4}{|l|}{$\log P$. ambiguus intensity\|l } \\
\hline Month & 26.4 & 11,280 & 0.01 \\
\hline Host sex & 1.6 & 1,280 & 0.2 \\
\hline Host sex ${ }^{\star} \log (x+1)$ M. pectinata & 9.3 & 1,280 & 0.00 \\
\hline Warren vegetation class & 16.2 & 1,280 & $<0.00$ \\
\hline Presence/absence of myxomatosis & 8.1 & 1,280 & 0.0 \\
\hline
\end{tabular}

All effects displayed were significant in the minimal model after bootstrapping (with the $P$ value set to 0.05 and iterations $=1,000$ ). d.f., degrees of freedom.

†The test statistic was the Wald Statistic $\left(\chi^{2}\right)$ for G. strigosum and M. pectinata, and the F Statistic for $T$. retortaeformis, $C$. denticulata and $P$. ambiguus.

$\ddagger$ Model type: REML (random terms $=$ warren number + year)

§Model type: REML (random term = warren number).

\|Model type: GLM.

that $T$. retortaeformis stimulates an acquired immune response whereas the G. strigosum numbers show no decline, suggesting no response to host immunity (Supplementary Note and Fig. S1). It is likely that an ability of G. strigosum, as seen in other helminths ${ }^{25}$, to modulate host immunity explains the positive effect this species appears to have on $T$. retortaeformis. Conversely, stimulation of acquired immunity by $T$. retortaeformis may negatively affect the related G. strigosum.

The interaction of $P$. ambiguus and $M$. pectinata is affected in both directions by host sex, once again implying mediation through host biology. Similarly, the negative effect of $M$. pectinata on upstream abundance of $G$. strigosum must be host mediated but the mechanism for these interactions is not discernible from the current data. The mechanism for the relationship between the cestode $C$. denticulata and the nematode $T$. retortaeformis cannot be determined, as the current data do not indicate whether the interaction is direct or host mediated.

These analyses provide credible evidence for the presence of interspecific parasite interactions throughout a community of gut parasites, suggesting that host immunity may have a role in shaping that community. Although we wish to stress that the predictions are based on analysis of correlated data and must be validated experimentally, the biology of the helminths provides support that the interactions are genuine.

Notably, there is no reason to believe that the rabbit system is unusual and therefore such analyses applied to other systems, particularly those of economically important livestock (where laboratory experiments have provided evidence of interaction ${ }^{21,26}$ ), are likely to reveal similar networks of interactions. The presence and detection of interspecific parasite interactions, particularly where they are immune mediated, could have important implications for parasite control strategies. This is particularly pertinent to the future of helminth control, where rapidly developing resistance to chemotherapeutics is leading to increased efforts in the field of vaccine development ${ }^{27}$. The work described here suggests that within-host interspecific parasite interactions may have a role in determining the overall impact of such vaccines on gut helminth communities. To illustrate this we used a simple generic model,

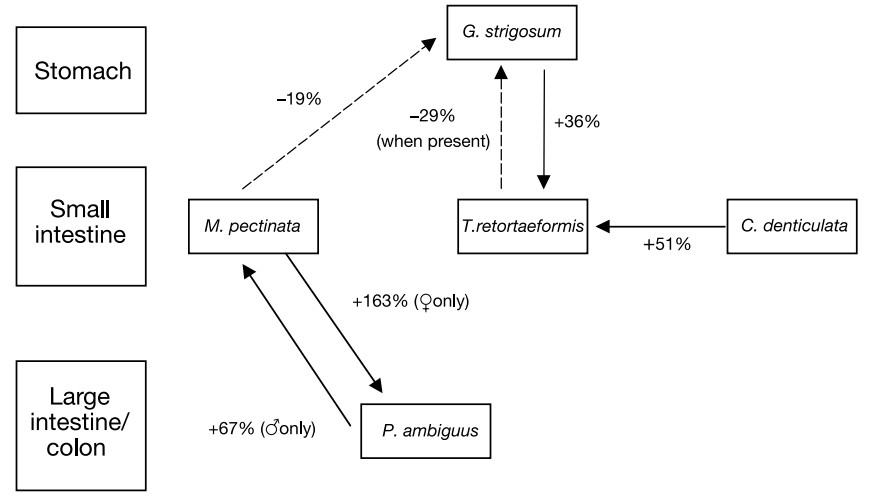

Figure 2 Relative positions of the rabbit gut helminths in the host gut, with interaction directions and strengths, based on observed mean burdens.

which may be adapted to represent any system. The model consisted of three parasite species within a single host (for details see Supplementary Methods). Each parasite stimulated a specific immune response at a rate proportional to the abundance of that parasite, and this immune response acted on the establishment rate of the parasite. Interspecific interactions were incorporated by allowing the immune response against one parasite to affect one of the other species, either increasing or suppressing the action of the immune response against that species. A simple network of interactions between the parasites was constructed such that parasite $1\left(\mathrm{P}_{1}\right)$ had a negative effect on parasite $2\left(\mathrm{P}_{2}\right), \mathrm{P}_{2}$ a positive effect on parasite $3\left(\mathrm{P}_{3}\right)$, and the effect of $\mathrm{P}_{3}$ on $\mathrm{P}_{1}$ (described by the parameter $\gamma_{3}$ ) was varied from highly negative to highly positive. A species-specific vaccine was then applied, targeted solely against $P_{1}$, and the impact of the strength of interaction between $\mathrm{P}_{3}$ and $\mathrm{P}_{1}$ on the efficacy of this vaccine was assessed.

Vaccination against the target parasite always suppressed its abundance below pre-treatment levels $\left(\Delta_{1}<1\right.$; Fig. 3b). However, vaccination against $\mathrm{P}_{1}$ tended to have a positive effect on $\mathrm{P}_{2}$ $\left(\Delta_{2}>1\right)$ (Fig. 3c), but little impact on $\mathrm{P}_{3}\left(\Delta_{3} \sim 1\right.$; Fig. $\left.3 \mathrm{~d}\right)$, presumably because $\mathrm{P}_{3}$ was two steps away from the target of the vaccine and was to some extent buffered from its effects.

When $\gamma_{3}$ was 0 (that is, $P_{3}$ did not interact with $P_{1}$ ), vaccination reduced $\mathrm{P}_{1}$ to approximately $15 \%$ of its pre-vaccination level (Fig. 3b). Furthermore, as $\mathrm{P}_{1}$ had a negative interaction with $\mathrm{P}_{2}$, its suppression meant that vaccination increased the abundance of $\mathrm{P}_{2}$ (Fig. 3c). When $\mathrm{P}_{3}$ had a strong negative interaction with $\mathrm{P}_{1}$ $\left(\gamma_{3}=-1\right)$ the vaccine, combined with this negative interaction, reduced $\mathrm{P}_{1}$ abundance to approximately $1 \%$ of its pre-vaccination level. As $\mathrm{P}_{1}$ was at very low abundance, its impact on $\mathrm{P}_{2}$ was negligible and so $\mathrm{P}_{2}$ approximated its pre-vaccination level $\left(\Delta_{2}\right.$ approached 1). However, when $\gamma_{3}$ was strongly positive $(+1) \mathrm{P}_{1}$ abundance was only reduced to about $50 \%$ of its pre-vaccination level; the positive impact of $\mathrm{P}_{3}$ on $\mathrm{P}_{1}$ diminished the efficacy of the vaccine. Notably, given a positive interaction between $P_{3}$ and $P_{1}$, vaccinating to control $P_{1}$ resulted in a massive relative increase in the abundance of $\mathrm{P}_{2}$, up to an order of magnitude greater than its pre-vaccination level. This is surprising because $P_{1}$ abundance is increased by the presence of $\mathrm{P}_{3}$ and so $\mathrm{P}_{2}$ abundance should be further suppressed due to the negative interaction between $\mathrm{P}_{1}$ and $\mathrm{P}_{2}$. The explanation for this counterintuitive result lies in the subtle nonlinear effects of the interactions. Before vaccination, the positive impact of $\mathrm{P}_{3}$ on $\mathrm{P}_{1}$ meant that $\mathrm{P}_{1}$ was at a very high equilibrium level. Hence, $\mathrm{P}_{2}$ was suppressed to a very low level. By applying the vaccine, $\mathrm{P}_{1}$ abundance was reduced to approximately half of its pre-vaccination level, thereby decreasing the negative impact on $\mathrm{P}_{2}$. $\mathrm{P}_{2}$ abundance therefore increased substantially, resulting in a massive relative increase compared with its pre-vaccination level. 

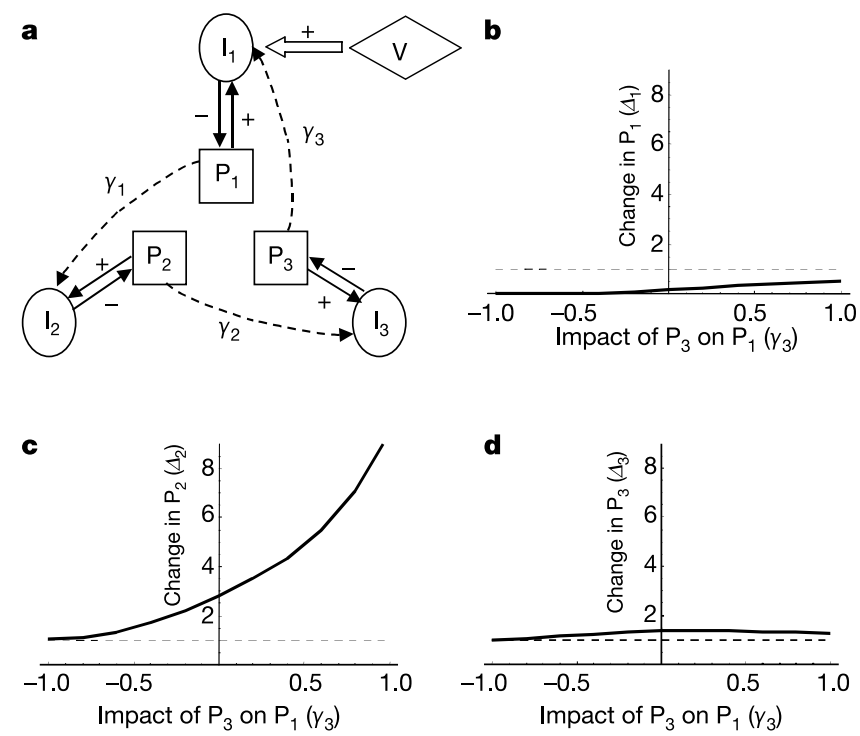

Figure 3 Three species parasite interaction model. a, Schematic of three parasite species interaction model (for details see Supplementary Methods) where $P_{1}, P_{2}$ and $P_{3}$ are the parasite species, $I_{1}, I_{2}$ and $l_{3}$ are the species-specific immune responses, $V$ is the vaccine applied against species 1 and $\gamma_{1}, \gamma_{2}$ and $\gamma_{3}$ are the parameters describing the strength of the effect of one species on another. $\mathbf{b}-\mathbf{d}$, Proportional change $\left(\Delta_{i}\right)$ in equilibrium abundances of parasite species $i$ where $i=1(\mathbf{b}), i=2(\mathbf{c})$ and $i=3(\mathbf{d})$, following vaccination (that is, post-vaccination equilibrium level/pre-vaccination equilibrium) at varying levels of $\gamma_{3}$, the parameter describing the strength of the effect of $P_{3}$ on $P_{1}$. The dotted horizontal line represents where the post-vaccination level $=$ pre-vaccination level.

Hence, vaccination against one parasite species produced unexpected and marked increases in the abundance of another, previously innocuous parasite species, by releasing it from the negative impact of the targeted parasite. These, and other, important effects would be missed if we ignored the possibility of such interactions.

The models used here have revealed an array of potential interactions between parasites, which would not have been elucidated by less powerful techniques. As concomitant infections are the norm rather than the exception ${ }^{28}$, application of these techniques to other mammalian-parasite data sets is likely to reveal similar interactions. Given the increase of anthelmintic resistance among gut helminths ${ }^{29}$, alternative control strategies are urgently needed. A clearer understanding of parasite community ecology may provide such an alternative. One implication is that parasites could be used to control other infections. For example, artificial infection with one relatively benign species could be used to stimulate cross-immunity and prevent subsequent infection with a deleterious species without resorting to anthelmintics. However, without the knowledge of the community relationships, treatment against one deleterious species could allow an increase in the intensity of a second harmful species. Previously unexplored interactions may be one explanation for the failure of laboratory proven vaccines to work under more natural conditions. It is clear that such interactions need to be incorporated into future dynamical studies of parasite communities where (with a few exceptions ${ }^{30}$ ) parasite interspecific conflicts and mutualisms are ignored in the null models.

\section{Methods}

Rabbits were collected from a 400 ha site in Perthshire, Scotland (ordnance grid reference NO 280340 ) between the months of January 1977 and December 1999. Total gut helminth counts, presence of myxomatosis and presence of Eimeria stiedae were recorded along with details of the external environment and aspects of host biology. Further details of the study site, collection protocols and data may be found in ref. 17 .

All analyses were conducted using the GENSTAT statistical package (VSN International Ltd) and all models were initially run as REMLs with the random terms of warren code and year of capture. Interactions between all model terms were initially included up to third order. Only adult rabbits (mass $>1,249 \mathrm{~g} ; n=1,526$ ) were used for this analysis because they were likely to have been exposed to infective stages of all parasites by this age. In addition, the distributions of the gut helminth parasites in different age classes of the rabbit have been shown to be different and must therefore be analysed separately ${ }^{17}$. The data for the gut helminths do not conform to the negative binomial distribution and in order to obtain a 'near' normal distribution for the dependent variable, zeros had to be removed from the data before a log transformation was applied ${ }^{17}$. We therefore considered those factors that affected the intensity of the species treated as the dependent variable (in infected animals only). Each helminth species in turn was treated as the dependent variable. The helminths not being treated as the dependent variable were included in the model (as determined from submodels) as independent factors (presence/absence) or as independent variables $(\log (x+1)$ intensity data) as appropriate. Nonsignificant terms were removed from the models in a stepwise manner until a minimal model was produced. This model was further refined following bootstrapping of the model effects and removal of any terms that were not significantly different from zero. Once the bootstrapping refinements were completed, each model was run once again in its final form to obtain effect sizes and significance levels. As model terms were only retained after the bootstrap of their effect size, $\log (x+1)$ transformation was considered sufficient for the species treated as independent variables and allowed comparison of concomitantly infected rabbits with those only infected with the species treated as the dependent variable.

Received 18 December 2003; accepted 10 March 2004; doi:10.1038/nature02490.

1. Behnke, J. M., Wakelin, D. \& Wilson, M. M. Trichinella spiralis: Delayed rejection in mice concurrently infected with Nematospiroides dubius. Exp. Parasitol. 46, 121-130 (1978).

2. Silver, B. B., Dick, T. A. \& Welch, H. E. Concurrent infections of Hymenolepsis diminuta and Trichinella spiralis in the rat intestine. J. Parasitol. 66, 786-791 (1980).

3. Christensen, N. O., Nansen, P., Fagbemi, B. O. \& Monrad, J. Heterologous antagonistic and synergistic interactions between helminths and between helminths and protozoans in concurrent experimentalinfection of mammalian hosts. Parasitol. Res. 73, 387-410 (1987).

4. Adams, D. B., Anderson, B. H. \& Windon, R. G. Cross-immunity between Haemonchus contortus and Trichostrongylus colubriformis in sheep. Int. J. Parasitol. 19, 717-722 (1989).

5. Behnke, J. M. et al. Variation in the helminth community structure in bank voles (Clethrionomys glareolus) from three comparable localities in the Mazury Lake District region of Poland. Parasitology 123, 401-414 (2001).

6. Cox, F. E. G. Concomitant infections, parasites and immune responses. Parasitology 122, S23-S38 (2001).

7. Bush, A. O. \& Holmes, J. C. Intestinal helminths of lesser scaup ducks: an interactive community. Can. J. Zool. 64, 142-152 (1986).

8. Haukisalmi, V. \& Henttonen, H. Coexistence in helminths of the bank vole Clethrionomys glareolus. 1. Patterns of cooccurrence. J. Anim. Ecol. 62, 221-229 (1993).

9. Haukisalmi, V. \& Henttonen, H. Coexistence in helminths of the bank vole Clethrionomys glareolus. 2. Intestinal distribution and interspecific interactions. J. Anim. Ecol. 62, 230-238 (1993).

10. Forbes, M., Weatherhead, P. J. \& Bennett, G. F. Blood parasites of blue grouse-Variation in prevalence and patterns of interspecific association. Oecologia 97, 520-525 (1994).

11. Poulin, R. Richness, nestedness, and randomness in parasite infracommunity structure. Oecologia 105, 545-551 (1996).

12. Poulin, R. Interactions between species and the structure of helminth communities. Parasitology 122, S3-S11 (2001).

13. Nilssen, A. C., Haugerud, R. E. \& Folstad, I. No interspecific covariation in intensities of macroparasites of reindeer, Rangifer tarandus (L.). Parasitology 117, 273-281 (1998).

14. Forbes, M. R., Alisauskas, R. T., McLaughlin, J. D. \& Cuddington, K. M. Explaining co-occurrence among helminth species of lesser snow geese (Chen caerulescens) during their winter and spring migration. Oecologia 120, 613-620 (1999).

15. Mead-Briggs, A. R. \& Vaughan, J. A. The incidence of anoplocephaline cestodes in a population of rabbits in Surrey, England. Parasitology 67, 351-364 (1973).

16. Mead-Briggs, A. R. \& Page, R. J. C. Records of anoplocephaline cestodes from wild rabbits and hares collected throughout Great Britain. J. Helminthol. 49, 49-56 (1975).

17. Boag, B., Lello, J., Fenton, A., Tompkins, D. M. \& Hudson, P. J. Patterns of parasite aggregation in the wild European rabbit (Oryctolagus cuniculus). Int. J. Parasitol. 31, 1421-1428 (2001).

18. Crofton, H. D. The ecology of the immature phases of trichostrongyle nematodes. v. The estimation of pasture infections. Parasitology 44, 313-324 (1954).

19. Boag, B. Similarities between the population dynamics of parasites of the wild rabbit and domestic animals. Asp. Appl. Biol. 53, 119-123 (1999).

20. Cowan, D. P. Aspects of the Behaviour of a Free-Living Population of the European Wild Rabbit, Oryctologus cuniculus L. in Southern England. Thesis, Royal Holloway College, Univ. London (1983).

21. Dobson, R. J. \& Barnes, E. H. Interaction between Ostertagia circumcincta and Haemonchus contortus infection in young lambs. Int. J. Parasitol. 25, 495-501 (1995).

22. Michel, J. F. Phenomenon of protection in infections of Trichostrongylus retortaeformis. Nature 172, 312 (1955).

23. Ford, G. E. Heterogeneity of allergens from, and homocytotropic antibody to, a gastro-intestinal nematode of rabbits. Immunology 21, 1073-1078 (1971).

24. Boag, B. \& Kolb, H. H. Influence of host age and sex on nematode populations in the wild rabbit (Oryctolagus cuniculus L.). Proc. Helminthol. Soc. Wash. 56, 116-119 (1989).

25. Behnke, J. M., Bajer, A., Sinski, E. \& Wakelin, D. Interactions involving intestinal nematodes of rodents experimental and field studies. Parasitology 122, S39-S49 (2001).

26. Christensen, C. M., Nansen, P. \& Barnes, E. H. The effect of concurrent or sequential Oesophagostomum dentatum and O. quadrispinulatum infections on the worm burdens of the two species in pigs. Parasitology 114, 273-278 (1997).

27. Dalton, J. P. et al. Helminth vaccines: from mining genomic information for vaccine targets to systems used for protein expression. Int. J. Parasitol. 33, 621-640 (2003).

28. Petney, T. N. \& Andrews, R. H. Multiparasite communities in animals and humans: frequency, structure and pathogenic significance. Int. J. Parasitol. 28, 377-393 (1998). 
29. Cornell, S. J., Isham, V. S., Smith, G. \& Grenfell, B. T. Spatial parasite transmission, drug resistance, and the spread of rare genes. Proc. Natl Acad. Sci. USA 100, 7401-7405 (2003).

30. Dobson, A. P. \& Roberts, M. The population dynamics of parasitic helminth communities. Parasitology 109, S97-S108 (1994).

Supplementary Information accompanies the paper on www.nature.com/nature.

Acknowledgements This work was undertaken at the University of Stirling and we thank the Natural Environment Research Council for funding the project. We also thank R. Norman of the University of Stirling for modelling assistance.

Competing interests statement The authors declare that they have no competing financial interests.

Correspondence and requests for materials should be addressed to J.L. (Joanne.Lello@csiro.au).

\section{Tug-of-war over reproduction in a social bee}

\section{Philipp Langer $^{1}$, Katja Hogendoorn ${ }^{2} \star \&$ Laurent Keller $^{1}$}

${ }^{1}$ Department of Ecology and Evolution, University of Lausanne, Biology Building, 1015 Lausanne, Switzerland

${ }^{2}$ School of Biological Sciences, Flinders University of South Australia, Adelaide SA 5001, Australia

* Present address: Centre for Plant and Pest Management, Adelaide University, Glen Osmond SA 5064, Australia

One of the main transitions in evolution is the shift from solitary organisms to societies with reproductive division of labour ${ }^{1,2}$. Understanding social evolution requires us to determine how ecological, social and genetic factors jointly influence group stability and partitioning of reproduction between group members $^{3-8}$. Here we test the role of the three key factors predicted to influence social evolution by experimentally manipulating them in a social allodapine bee. We show that increased relatedness between nestmates results in more even reproduction among group members and a greater productivity per individual. By contrast, the degree of reproductive skew is not influenced by the opportunity for solitary breeding or by the potential benefits of cooperation. Relatedness also has a positive effect on group stability and overall productivity. These findings are in line with predictions of the tug-of-war models, in which the degree of reproductive division of labour is determined primarily by selfish competition between group members. The alternative view, where the degree of reproductive skew is the outcome of a social contract between potential breeders, was not supported by the data.

Reproductive skew models can be divided into two classes on the basis of the assumed power relations in a social group. In transactional models, group members yield reproduction to each other in return for specific benefits, whereas in tug-of-war models reproductive sharing reflects each group member's inability to effectively monopolize reproduction $^{5,6}$ (Table 1). The first of the two main transactional models, the concession model, assumes that a dominant individual fully controls both group membership and the fraction of total group reproduction that a subordinate obtains ${ }^{9-11}$. Under this model, the dominant yields just enough reproduction to a subordinate to make it worthwhile for the subordinate to stay and cooperate peacefully rather than leave the group to reproduce solitarily. This model predicts that reproductive skew increases with higher genetic relatedness between the dominant and the subordinate $(r)$, and higher overall reproductive output of a group of two breeders relative to the output of a single dominant $(k)$, but decreases with higher expected solitary reproduction of a potential subordinate relative to that of a lone dominant $(x$, lower values of $x$ indicating harsher ecological constraints on solitary breeding). This is because the subordinate gains greater fitness benefits from cooperation with increased $r$ and $k$, and thus requires less direct reproduction. However, when the chances of successful solitary breeding of the subordinate are high (increased $x$ ), the dominant has to concede a larger fraction of colony reproduction to the subordinate to make staying in the group profitable for the latter. The other main transactional model, the restraint model, assumes that the dominant controls group membership but that a subordinate fully controls its reproductive share within the group ${ }^{12}$. Under this model, the subordinate captures the largest share of reproduction that the dominant will tolerate before ejecting the subordinate, resulting in opposite predictions to the concession model (Table 1). Finally, tug-of-war models assume that both dominant and subordinate individuals have only limited control over the allocation of reproduction and must expend effort to increase their shares of the total group output. The main prediction of this model is that reproductive skew is negatively correlated with or independent of relatedness because struggle over reproduction takes place at the cost of overall group productivity, with the effect that higher relatedness acts as a break on the investment in reproductive competition ${ }^{13}$. This model predicts that skew is not correlated with group productivity $(k)$ or the ecological constraint on solitary breeding $(x)$. Unlike the two transactional models, where relatedness has no influence on group productivity, the tug-of-war model predicts a positive correlation between breeder relatedness and total group output, because related females allocate less energy to reproductive competition.

We tested the predictions of reproductive skew models in the Australian allodapine bee Exoneura nigrescens by experimentally manipulating all three parameters $(r, x$ and $k)$. Several characteristics make this species an ideal experimental system. First, cooperation is facultative, with females nesting either solitarily or with up to three other females ${ }^{14}$, which allows direct comparison between social and solitary productivities $(k)$. Second, there are no morphological castes, hence all females have the potential to reproduce $^{11}$. Third, a high proportion $(51 \%)$ of multi-female nests contain two females ${ }^{15}$, the type of groups for which most reproductive skew models have been developed. Fourth, withinnest relatedness $(r)$ is variable $\mathrm{e}^{14,16,17}$ and can be experimentally manipulated. Fifth, nests are built primarily in dead dried flower stalks of grasstrees (Xanthorrhoea minor) and Melaleuca squarrosa ${ }^{15}$,

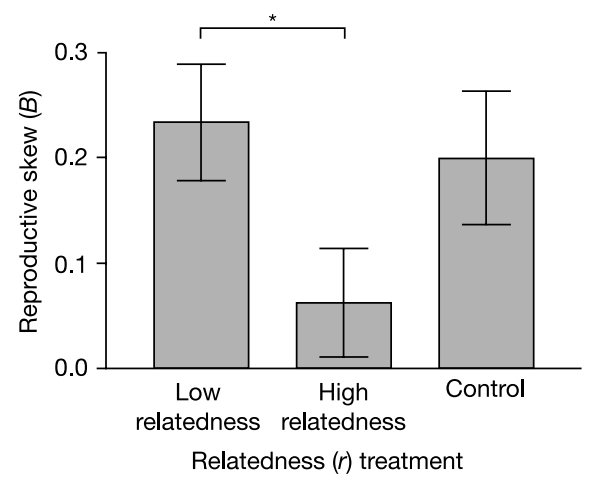

Figure 1 Reproductive skew in nests of the high- and low-relatedness treatment, and in unmanipulated control nests. Overall skew was significantly greater than zero in both the low-relatedness treatment (one-sample $t$-test, $t=4.2$, d.f. $=6, P=0.005$ ) and natural nests $(t=3.2$, d.f. $=8, P=0.01)$. In the high-relatedness treatment, however, skew was not significantly different from zero $(t=1.2$, d.f. $=12, P=0.24)$, the expected value if breeders share reproduction equally ${ }^{29}$. Error bars indicate standard errors, $n=29$. 